ISSUES IN MEDICINE

\title{
Medicine-use evaluation of doxazosin prescribing to inform formulary recommendations
}

\author{
A Wehmeyer, ${ }^{1}$ BPharm; R Coetzee, ${ }^{1}$ BPharm, MPharm, PharmD; N Hoffman, ${ }^{1}$ BPharm; Y Johnson, ${ }^{2}$ BPharm, MPH; \\ R Kloppers, ${ }^{3}$ BPharm, MPharm \\ ${ }^{1}$ Faculty of Natural Sciences, School of Pharmacy, University of the Western Cape, Cape Town, South Africa \\ ${ }^{2}$ Pharmacy Services, Western Cape Government Health, Cape Town, South Africa \\ ${ }^{3}$ Faculty of Pharmacy, Rhodes University, Grahamstown, South Africa
}

Corresponding author: $R$ Coetzee (recoetzee@uwc.ac.za)

Background. It is estimated that $>50 \%$ of all medicines are inappropriately prescribed, dispensed or sold. In 2018, the Western Cape Government Health (WCGH) Provincial Pharmacy and Therapeutics Committee (PPTC) endorsed an investigation of doxazosin use in provincial public sector healthcare facilities using a medicine-use evaluation (MUE). This was based on potential inappropriate prescribing, high usage and high costs associated with doxazosin.

Objectives. To determine whether doxazosin was being prescribed in accordance with recommendations from standard treatment guidelines and essential medicines lists for hospital and primary healthcare level, as well as from the WCGH provincial code list.

Methods. The study design consisted of a retrospective multicentre prescription review. The study population included all adult outpatients who were prescribed doxazosin, which was to be dispensed by the WCGH Chronic Dispensing Unit. Criteria for evaluation included indications for doxazosin administration and total daily dose prescribed.

Results. The study sample comprised 171 patients. The main indication for doxazosin administration was as a fourth-line agent for the treatment of hypertension (82\%). The remaining indications were not guideline approved (18\%). The total daily doses identified were $4 \mathrm{mg}$ (62\%), followed by $8 \mathrm{mg}(36 \%)$ and $2 \mathrm{mg}(2 \%)$. All of these were approved dosages as per guideline recommendations.

Conclusions. MUEs are an untapped resource for medication-use monitoring and practice improvement. MUEs offer a distinct, costeffective approach to improve medicine use in all settings. This MUE revealed a relatively minor concern with doxazosin prescribing. MUEs can be beneficial to inform formulary changes and re-evaluate medication-use restrictions.

S Afr Med J 2020;110(1):16-20. https://doi.org/10.7196/SAMJ.2020.v110i1.14295

The World Health Organization (WHO) estimates that $>50 \%$ of all medicines are inappropriately prescribed, dispensed or sold. Together with the global estimate that half of all patients do not use medicines appropriately, this constitutes a significant wastage of limited resources. ${ }^{[1]}$ Irrational medicine use is a significant problem in developing countries, as such countries have poorly functioning health systems where mechanisms for routine monitoring of medication use are underdeveloped or entirely absent. ${ }^{[2]}$

Medicine-use evaluation (MUE) is an effective tool that may be used to overcome irrational medicine use. It is a type of qualityimprovement strategy that employs an ongoing, systematic, criteriabased approach to evaluate medicine use and ensure optimal patient outcomes. ${ }^{[3,4]}$ MUEs follow a quality-improvement approach, where the primary function is to analyse practice performance and highlight areas for improvement. ${ }^{[5]}$

The main objectives of MUEs are to improve and standardise medication-use processes, promote cost-effective therapy and ensure patient safety. Various categories of medicines and/or medicinerelated events can serve as indicators of possible concerns in the medication-use process, and are therefore employed to identify target areas for MUEs. ${ }^{[3]}$ These include medicines that are associated with inappropriate use, high-cost medicines, medicines or medication-use processes where suboptimal administration is expected to negatively impact patient outcomes, medicines that are potentially toxic at standard doses and medicines that are being considered for retention in, as an addition to or for deletion from a formulary. ${ }^{[3,4,6]}$
Doxazosin is a selective $\alpha 1$-adrenergic receptor antagonist, which induces vasodilation and reduces peripheral vascular resistance. It is typically used as part of multidrug regimens in the treatment of hypertension - more specifically resistant hypertension. It is also used for the treatment of lower-urinary tract symptoms related to benign prostatic hyperplasia. ${ }^{[7,8]}$ Doxazosin is not recommended as a first-line antihypertensive agent, which is primarily related to the findings of the Antihypertensive and Lipid-Lowering Treatment to Prevent Heart Attack Trial (ALLHAT). ALLHAT revealed that doxazosin had an inferior antihypertensive effect compared with other agents, which led to various guidelines recommending against doxazosin monotherapy in hypertension. The trial also showed that doxazosin was associated with a higher risk of stroke and heart failure than chlorthalidone in high-risk hypertensive patients. ${ }^{[9,10]}$

Doxazosin use as a fourth-line agent in resistant hypertension, where $\geq 4$ antihypertensive agents are required to achieve normotension, is decreasing. ${ }^{[8,11]}$ This has occurred primarily in response to the findings of the PATHWAY-2 (spironolactone v. placebo, bisoprolol, and doxazosin to determine the optimal treatment for drug-resistant hypertension) randomised controlled trial, which demonstrated the superior efficacy of spironolactone as a fourth-line agent in resistant hypertension when compared with doxazosin and bisoprolol. ${ }^{[12]}$ This finding has been supported by larger meta-analyses and systematic reviews. ${ }^{[11,13]}$

The use of doxazosin is restricted in the Western Cape Province's public healthcare sector, where it is only recommended for treating 
phaeochromocytoma ${ }^{[14,15]}$ and as a fourth-line antihypertensive agent. ${ }^{[16]}$ In accordance with Western Cape Government Health (WCGH) policy, doxazosin initiation was restricted to specialist physicians and paediatricians. In 2018, an application was submitted to the WCGH Provincial Pharmacy and Therapeutics Committee (PPTC) requesting that the restriction be reduced to allow family physicians to initiate doxazosin at a primary healthcare (PHC) level. For this request to be considered, the PPTC needed to ascertain whether doxazosin was being used appropriately, as its usage had increased with time. Therefore, the PPTC endorsed a recommendation to investigate doxazosin (standard formulation) use in the Western Cape. This investigation was to be conducted in all provincial public sector healthcare facilities through the use of an MUE. Data from the WCGH Medical Depot (CMD) revealed that doxazosin was widely used in PHC facilities, where 247918 packs of $4 \mathrm{mg}$ tablets (30s) were issued from 1 August 2017 to 31 July 2018. The total cost of these purchases was calculated at ZAR6 744 995. Therefore, concerns regarding inappropriate prescribing, together with high use (in relation to doxazosin's restricted use) and high cost, had been recognised.

\section{Methods}

The design comprised a retrospective, multicentre prescription review. This review was intended to inform on a potential adoption of an MUE at all provincial public sector facilities.

The study population included urban and rural adult outpatients ( $\geq 18$ years of age) who were prescribed doxazosin, which was dispensed by the WCGH Chronic Dispensing Unit (CDU) from 1 August to 31 December 2018. Patients receiving medicines from the CDU are stabilised on maintenance doses of all of their treatments, including doxazosin. Therefore, patients who had not been stabilised on maintenance doses of doxazosin were excluded from the study.

The estimated prevalence of resistant hypertension in South Africa (SA) is $12.1 \% \cdot{ }^{[17]}$ Based on this prevalence, a sample of 163 prescriptions was required.

Information was collected retrospectively from electronic CDU prescriptions. Electronic copies of prescriptions were randomly selected from the CDU database using random systematic selection. This was conducted through the randomisation of a list of patient folder numbers, followed by patient selection at fixed, periodic intervals. The corresponding electronic prescriptions were then retrieved from the CDU database. Data from electronic prescriptions were recorded with the use of a predesigned and piloted data collection tool. Information collected was captured onto a predesigned Microsoft Office Excel (Microsoft, USA) spreadsheet, from where analyses were performed.

MUE criteria were established in accordance with recommendations provided by the SA Standard Treatment Guidelines (STG) and Essential Medicines List (EML) for PHC level (2014), ${ }^{[14]}$ adult hospital level (2015), ${ }^{[15]}$ as well as the WCGH Provincial Code List (2018). ${ }^{[16]}$ The Code List, which functions as a formulary, was released by the WCGH to provide guidance on the use of doxazosin $1 \mathrm{mg}$ tablets in the Western Cape. It also provides information on prescribing restrictions related to the use of $4 \mathrm{mg}$ doxazosin tablets as a fourth-line antihypertensive agent - only specialist physicians and paediatricians are allowed to prescribe it. Table 1 provides approved indications and dosages for doxazosin in accordance with the aforementioned guidelines and Code List.

Target thresholds for adherence to guideline recommendations for indication and dosage were set at $95 \%$. As doxazosin was prescribed predominantly at PHC level, 95\% of all identified prescriptions should have adhered to the requirements set out by the relevant guidelines. Therefore, a deviation of $5 \%$ from these requirements was set for outliers that were deemed appropriate owing to patientspecific factors, such as medicine allergies.

\section{Ethical approval}

Approval to conduct the study was granted by the WCGH PPTC, as well as the University of the Western Cape Biomedical Research Ethics Committee (ref. no. BM18/4/5).

\section{Results}

The sample consisted of 171 prescriptions, which were randomly selected from 8080 prescriptions that were available on the WCGH $\mathrm{CDU}$ database. There were approximately twice as many females as males $(68 \%$ v. $32 \%)$. The mean age of patients comprising the total sample was 61 years - gender and age are given in Table 2 .

The type of healthcare facility where doxazosin was prescribed was also investigated. Community health centres (CHCs), clinics and community day centres (CDCs) comprised $36 \%, 37 \%$ and $21 \%$ of the total sample, respectively. These equate to a total contribution of $94 \%$ to the total sample, while the remaining $6 \%$ consisted of district hospitals.

The proportion of the total sample by district/substructure was also investigated. Facilities from rural areas comprised $58 \%$ of the total sample, while the remaining $42 \%$ consisted of facilities located

Table 1. Criteria for evaluation

South African STGs and EML for PHC level ${ }^{[14]}$ and adult hospital level ${ }^{[15]}$ Indication

Total daily dosage, mg

Phaeochromocytoma

$1-16$

WCGH Provincial Code List ${ }^{[16]}$

Indication

Total daily dosage, mg

Hypertension (fourth-line therapy)

$1-8$

$\mathrm{STG}=$ Standard Treatment Guidelines; $\mathrm{EML}=$ Essential Medicines List $; \mathrm{PHC}=$ primary healthcare; $\mathrm{WCGH}=$ Western Cape Government Health

Table 2. Demographics of patient sample $(N=171)$

\begin{tabular}{llllll}
\hline Gender & Patients, $\boldsymbol{n}$ & Sample, $\%$ & Mean age, years $(\mathrm{SD})$ & Minimum age, years & Maximum age, years \\
\hline Female & 116 & 68 & $60(10.6)$ & 37 & 89 \\
Male & 55 & 32 & $61(10.0)$ & 43 & 80 \\
Total & 171 & 100 & $61(10.4)$ & 37 & 89 \\
SD = standard deviation. & & & & &
\end{tabular}


in the Cape Metropole. The Cape Winelands, Eden and SouthWestern districts contributed the most to the proportion of the total sample $-23 \%, 16 \%$ and 15\%, respectively. The Eastern/Khayelitsha, Northern/Tygerberg, Klipfontein/Mitchell's Plain and Central Karoo districts comprised $13 \%, 10 \%, 4 \%$ and $1 \%$ of the total sample, respectively. Both the Overberg and West Coast districts comprised $9 \%$ of the total sample.

A total of 179 indications were observed from the 171 doxazosin prescriptions included in the sample. Seven patients used doxazosin for both hypertension and benign prostatic hyperplasia, while 1 patient used doxazosin for both hypertension and unconfirmed urinary incontinence. The predominant indication for doxazosin was as a fourth-line agent for the treatment of hypertension (i.e. doxazosin was prescribed concurrently with a minimum of 3 other antihypertensive medications as per the Code List). ${ }^{[16]}$ The remaining indications for doxazosin contributed to $18 \%$ of the proportion of the total sample. These indications included hypertension, where doxazosin was prescribed as a first-, second- or third-line agent, and constituted $13 \%$ of the total sample. Doxazosin indications for benign prostatic hyperplasia were also evident from the findings and made up $4 \%$ of the sample. No cases of phaeochromocytoma were observed in this sample. Fig. 1 displays the proportion of the various indications identified for doxazosin use.

The most predominantly identified total daily dose for doxazosin was $4 \mathrm{mg}$, which constituted $62 \%$ of the total sample. This was followed by $8 \mathrm{mg}$, which constituted $36 \%$ of the total sample, while $2 \mathrm{mg}$ constituted only $2 \%$ of the total sample. No total daily doses of $>16 \mathrm{mg}, 16 \mathrm{mg}$ or $1 \mathrm{mg}$ of doxazosin were observed. Fig. 2 presents the proportions of total daily doses of doxazosin identified from the sample.

A total of 579 antihypertensive agents were used in conjunction with doxazosin, which equates to an average of 3 additional antihypertensive agents (Table 3 ). The most frequently used additional antihypertensive agent was amlodipine, which contributed $25 \%$ to the total usage. This was followed by enalapril and atenolol, which each made up $20 \%$ of the proportion of the total sample, respectively.

\section{Discussion}

As the mean age of patients in the sample was 61 years, it can be construed that these patients were predominantly elderly. This

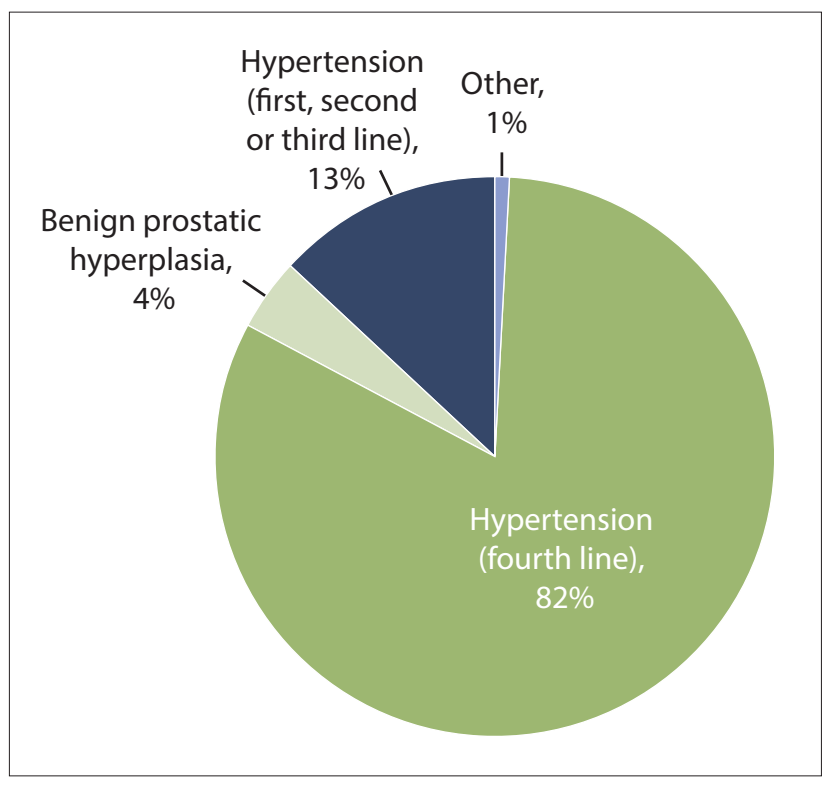

Fig. 1. Proportion of total sample by indication $(\mathrm{N}=179)$. finding was anticipated, as the risk of developing hypertension is directly correlated with increasing age. ${ }^{[18]}$ As the prevalence of resistant hypertension is more frequently observed in persons aged $\geq 60$ years, ${ }^{[19]}$ the age statistic of our sample is comparable. As the sample consisted primarily of female patients, a correlation between gender and potentially resistant hypertension was not ascertainable.

The finding that $\mathrm{PHC}$ facilities, including $\mathrm{CHCs}$, $\mathrm{CDCs}$ and clinics, comprised $94 \%$ of the total sample, was anticipated. This distribution was predicted, as prescriptions were identified from the Western Cape's CDU database, which consists of prescriptions from patients who are stable while taking their chronic medications and are typically down-referred to PHC facilities.

The finding that rural districts consisted of a larger proportion of the total sample (58\%), was unexpected. However, doxazosin consumption data from January to March 2018 showed that 10139 packs of $4 \mathrm{mg}$ doxazosin tablets (30s) were used by the top 10 consuming facilities in the Western Cape. Of the 10139 packs issued, rural facilities used 7230 packs, while the remaining 2909 packs were used by Cape Metropole facilities. Furthermore, during this period, 6 of these top 10 consuming facilities were rural facilities. Therefore, it can be inferred that the finding of this MUE, where rural facilities were the highest consumers of doxazosin, was appropriate.

Doxazosin was administered as a fourth-line agent for the treatment of hypertension, which was the most predominant indication identified - an approved indication as per the relevant

Table 3. Concurrent antihypertensive agents used in the sample $(N=579)$

\begin{tabular}{ll}
\hline $\begin{array}{l}\text { Antihypertensive agent } \\
\text { prescribed }\end{array}$ & $\boldsymbol{n}(\%)$ \\
\hline Hydrochlorothiazide & $95(16)$ \\
Enalapril & $116(20)$ \\
Amlodipine & $142(25)$ \\
Losartan & $22(4)$ \\
Furosemide & $60(10)$ \\
Atenolol & $114(20)$ \\
Spironolactone & $24(4)$ \\
Carvedilol & $6(1)$ \\
Total & $579(100)$
\end{tabular}



Fig. 2. Proportion of total daily doses of doxazosin prescribed. 
circular. ${ }^{[16]}$ The lack of doxazosin use in phaeochromocytoma, which was the only STG- and EML-approved indication for doxazosin, can be attributed to the low estimated prevalence of the disease $\left(0.5 \%\right.$ among those tested) ${ }^{[20]}$ Doxazosin's relatively low usage as a first-, second- or third-line antihypertensive agent indicated that prescribers predominantly followed the recommendations of the relevant guidelines and circular; however, doxazosin in these scenarios is unusual in the public sector and would require further investigation. These cases can potentially be attributed to patients who were intolerant to other antihypertensive agents, who were allergic to the other agents or who did not respond well to the use of other agents. Moreover, the identified low use of doxazosin in benign prostatic hyperplasia can be based on similar factors relative to its use.

The recommended total daily dosing range for doxazosin as per the relevant circular is $1-8 \mathrm{mg} \cdot{ }^{[16]}$ As the $4 \mathrm{mg}$ total daily dose was mostly identified in this study, it can be construed that prescribers followed the set recommendations rigorously. This was followed by the $8 \mathrm{mg}$ total daily dose. The recommended initiation dose of doxazosin in hypertension is $1 \mathrm{mg}$ daily. ${ }^{[21]}$ Therefore, the findings of this study indicate that the majority in the sample were stable chronic hypertensive patients. This result was anticipated, as the prescriptions were collected from the CDU, where patients who are classified as stable while receiving their chronic regimens have such medications prepacked. Prescriptions submitted to the CDU should be for patients who have been stabilised on a maintenance dose before referral. The study showed that most patients received $4 \mathrm{mg}$ daily (62\%), followed by $8 \mathrm{mg}$ daily (36\%), which is in line with recommended maintenance doses.

The average number of concurrently prescribed antihypertensive agents was 3 per patient, which indicates that doxazosin was used predominantly in combination with at least 3 other agents. Doxazosin was therefore most likely used as a fourth-line agent for treating hypertension. However, as amlodipine (25\%), enalapril (20\%) and atenolol (20\%) were the most co-prescribed agents, it can be inferred that adherence to the STG and EML stepwise approach to treating hypertension was lacking. In terms of medicine therapy, this stepwise approach recommends the use of hydrochlorothiazide initially, followed by the addition of either an angiotensin-converting enzyme (ACE) inhibitor or a long-acting calcium channel blocker. ${ }^{[1,15]}$ Therefore, a more appropriate finding would have been greater use of hydrochlorothiazide, which comprised only $16 \%$ of the total sample. The prescribing of doxazosin fell 13\% short of the approved indication threshold, which was set at $95 \%$. Although this deficit may be interpreted as being marginal, recommendations to enhance practice performance should be encouraged. Therefore, several recommendations have been outlined.

Pharmacy staff should be encouraged to be vigilant of all prescriptions for doxazosin and should note the indications listed. If any unapproved indications are present, they should query the indication with the relevant prescriber, who should be a specialist physician or a paediatrician. To ensure the support of pharmacy staff in this regard, a circular describing the need to review all indications listed for doxazosin on prescriptions can be distributed.

Healthcare facilities can implement a monthly or bi-monthly monitoring strategy for doxazosin use. This strategy should entail the evaluation of issues and/or purchases of doxazosin to assess prescribing trends and increased consumption. This strategy has the potential to inform future MUEs and target areas for practice improvement.
The WCGH PPTC should consider endorsing a follow-up provincewide MUE for doxazosin. This should be implemented in every public sector healthcare facility, where a retrospective folder review can be conducted. It will allow facility staff to gauge their adherence to the set recommendations and encourage them to improve prescribing behaviours with regard to doxazosin usage.

As the threshold deficit was relatively low, the PPTC and other stakeholders should consider reducing prescribing restrictions on doxazosin use. This would allow more patients to benefit from the medication and encourage prescribers to adhere to set recommendations. However, further strategies to improve doxazosin monitoring should be implemented concurrently with a reduction in restrictions.

\section{Conclusions}

MUEs are an unexploited resource for medication-use monitoring and practice improvement in developing countries. They offer a unique, cost-effective system to improve medicine use in any setting. As this MUE revealed a relatively minor concern with doxazosin prescribing, MUEs may also inform formulary changes and influence policy. This was demonstrated by the finding of this MUE, where inappropriate prescribing of doxazosin was ascertained to be only $13 \%$. In light of this, the WCGH PPTC amended the policy regarding doxazosin prescription restrictions, where it would permit family physicians to prescribe the medication. Follow-up MUEs and other strategies to improve medicine use should be conducted after an initial MUE to ensure continuous practice evaluation and improvement. MUE success is founded on multidisciplinary participation and organisation, which must oversee continuous cycles of practice evaluation, intervention and change. Opportunities for the use of MUEs should be systematically identified and form a routine part of clinical practice moving forward.

Declaration. This article was completed in accordance with the requirements for AW's M Clin Pharm degree.

Acknowledgements. We wish to acknowledge and thank the WCGH PPTC for their endorsement of the study. We would also like to thank the CDU for their aid in accessing the electronic prescription database and for compiling a list of all patients who had been prescribed doxazosin at the time of data collection.

Author contributions. AW: conceptualised and designed the study, performed data collection and analysis and drafted the manuscript; RC: supervised the study, helped to design the study and data collection tools, and was a major contributor to the final manuscript; NH, RK,YJ: contributed to the final manuscript.

Funding. None.

Conflicts of interest. None.

\footnotetext{
1. World Health Organization. The world medicines situation. 2011. http://apps.who.int/medicinedocs/ en/d/Js6160e/10.html (accessed 28 March 2019).

Ofori-Asenso R, Brhlikova P. Pollock AM. Prescribing indicators at primary health care centers within the WHO African region: A systematic analysis (1995 - 2015). BMC Public Health 2016;16:724. https://doi.org/10.1186/s12889-016-3428-8

3. SHPA Committee of Specialty Practice in Drug Use Evaluation. SHPA standards of practice for drug use evaluation in Australian hospitals. J Pharm Pract Res 2004;34(3):220-223. https://doi.org/10.1002/ jppr2004343220

4. Management Sciences for Health and World Health Organization. Drug and therapeutics committee training course. https://www.who.int/medicines/technical_briefing/tbs/Participant-s-Guide-All-Sessions. pdf (accessed 28 March 2019).

5arkey P, Reller MK, Resar RK. Basics of quality improvement in health care. Mayo Clinic Proc 2007;82(6):735-739. https://doi.org/10.4065/82.6.735

6. American Society of Health-system Pharmacists. ASHP guidelines on medication-use evaluation. Am J Health System Pharmacists 1996;53(16):1953-1955. https://doi.org/10.1093/ajhp/53.16.1953
} 
7. Gaelzer MM, Coelho BP, de Quadros AH, et al. Phosphatidylinositol 3-kinase/AKT pathway inhibition by doxazosin promotes glioblastoma cells death, upregulation of p53 and triggers low neurotoxicity. PLoS ONE 2016;11(4):e0154612. https://doi.org/10.1371/journal.pone.0154612

8. Pio-Abreu A, Drager LF. Resistant hypertension: Time to consider the best fifth anti-hypertensive treatment. Curr Hypertens Rep 2018;20(8):67. https://doi.org/10.1007/s11906-018-0866-y

9. Wykretowicz A, Guzik P, Wysocki H. Doxazosin in the current treatment of hypertension. Expert Opin Pharmacother 2008;9(4):625-633. https://doi.org/10.1517/14656566.9.4.625

10. ALLHAT Officers and Coordinators for the ALLHAT Collaborative Research Group. Major outcomes in high-risk hypertensive patients randomized to angiotensin-converting enzyme inhibitor or calcium channel blocker vs. diuretic: The Antihypertensive and Lipid-Lowering Treatment to Prevent Heart Attack Trial (ALLHAT). JAMA 2002;288(23):2981-2997. https://doi.org/10.1001/jama.288.23.2981

11. Sinnott SJ, Tomlinson LA, Root AA, et al. Comparative effectiveness of fourth-line anti-hypertensive agents in resistant hypertension: A systematic review and meta-analysis. Eur J Prevent Cardio 2017;24(3):228-238. https://doi.org/10.1177/2047487316675194

12. Williams B, MacDonald TM, Morant S, et al. Spironolactone versus placebo, bisoprolol, and doxazosin to determine the optimal treatment for drug-resistant hypertension (PATHWAY-2): A randomised, to determine the optimal treatment for drug-resistant hypertension (PATHWAY-2): A randomised,
double-blind, crossover trial. Lancet 2015;386(10008):2059-2068. https://doi.org/10.1016/S01406736(15)00257-3

13. Tataru AP, Barry AR. A systematic review of add-on pharmacologic therapy in the treatment of resistant hypertension. Am J Cardiovasc Drugs 2017;17(4):311-318. https://doi.org/10.1007/s40256-017-0224-5

14. National Department of Health. Standard Treatment Guidelines and Essential Medicines List. Primary Healthcare. Pretoria: Government Printer, 2014
15. National Department of Health. Standard Treatment Guidelines and Essential Medicines List. Hospital Level. Adults. Pretoria: Government Printer, 2015.

16. Western Cape Government Health. Provincial Code List. Cape Town: Government Printer, 2018

17. Nansseu JR, Nansseu JRN, Noubiap JJN, et al. The highly neglected burden of resistant hypertension in Africa: A systematic review and meta-analysis. BMJ Open 2016;6:e011452. https://doi.org/10.1136 in Africa: A systematic

18. Bradshaw D, Steyn K, Levitt N, Nojilana B. Non-communicable diseases - a race against time, 2011 http://www.health.uct.ac.za/usr/health/research/groupings/cdia/downloads/MRC_policy_brief.pdf (accessed 23 March 2019)

19. Achelrod D, Wenzel U, Frey S. Systematic review and meta-analysis of the prevalence of resistan hypertension in treated hypertensive populations. Am J Hypertens 2015;28(3):355-361. https://doi org/10.1093/ajh/hpul51

20. Pacak K, Linehan WM, Eisenhofer G, Walther MM, Goldstein DS. Recent advances in genetics, diagnosis, localization, and treatment of pheochromocytoma. Ann Intern Med 2001;134(4):315-329. https://doi.org/10.7326/0003-4819-134-4-200102200-00016

21. Chung N, Vashi V, Puente J, Sweeney M, Meredith P. Clinical pharmacokinetics of doxazosin in controlled-release gastrointestinal therapeutic system (GITS) formulation. Br J Clin Pharmacol 1999;48(5):678-687. https://doi.org/10.1046\%2Fj.1365-2125.1999.00067.x

Accepted 10 September 2019. 